\title{
Síndrome de la arteria mesentérica superior en una adolescente de 12 años. Caso clínico
} Superior mesenteric artery syndrome in a 12-year-old adolescent. Clinical case

\author{
Dra. Graciela S. Menalled ${ }^{a}$, Dr. Héctor Colombo ${ }^{a}$, Dra. Susana Montero ${ }^{a}$ y Dra. Laura del V. Poeta Casalis ${ }^{a}$
}

\section{RESUMEN}

El síndrome de la arteria mesentérica superior es una enfermedad poco frecuente en pediatría. Se produce por la compresión de la tercera porción duodenal a su paso entre la arteria mesentérica superior y la aorta abdominal (compás aortomesentérico). La mayoría de los pacientes presentan factores predisponentes: pérdida de peso aguda o compresiones extraabdominales.

Se presenta el caso de una niña de 12 años de edad a quien se le diagnosticó el síndrome sin presentar factores predisponentes. Comenzó de modo súbito con náuseas, vómitos incoercibles y dolor abdominal, que era posprandial y se aliviaba, llamativamente, en decúbito lateral izquierdo. Esto constituyó la sospecha clínica del síndrome, por lo que se solicitó una angio tomografía computada abdominal y se observó el estrechamiento del compás aortomesentérico. Se realizó un tratamiento médico conservador, sin respuesta clínica. Se decidió el tratamiento quirúrgico y se logró la resolución del cuadro clínico.

Palabras clave: obstrucción duodenal, sindrome de la arteria mesentérica superior.

\begin{abstract}
The superior mesenteric artery syndrome is rarely seen in children. It results from an intestinal obstruction due to compression of the third portion of duodenum between the superior mesenteric artery and the abdominal aorta. In most of the cases there are predisposing factors such as rapid weight loss or extra-abdominal compression.

We report a case of a superior mesenteric artery syndrome in a twelve-year-old female patient without predisposing factors. The girl began suddenly with nauseas, continuous vomiting and abdominal pain. The abdominal pain was postprandial and it decreased in left lateral decubitus position. Clinically, this characteristic suggested superior mesenteric artery syndrome. Angio-computed tomography scan confirmed the diagnosis. Given that conservative treatment ultimately failed, patient was subjected to surgery and the illness was resolved.

Key words: duodenal obstruction, superior mesenteric artery syndrome.
\end{abstract}

a. Hospital de Niños Santísima Trinidad, Córdoba, Argentina.

\author{
Correspondencia: \\ Dra. Graciela S. Menalled: graciela.menalled@gmail.com \\ Financiamiento: Ninguno.
}

Conflicto de intereses: Ninguno que declarar.

Recibido: 30-11-2018

Aceptado: 11-6-2019 http: / / dx.doi.org/10.5546/aap.2019.e648

Cómo citar: Menalled GS, Colombo H, Montero S, Poeta Casalis L. Síndrome de la arteria mesentérica superior en una adolescente de 12 años. Caso clínico. Arch Argent Pediatr 2019;117(6):e648-e650.

\section{INTRODUCCIÓN}

El síndrome de la arteria mesentérica superior (SAMS) es una causa poco frecuente en pediatría (el 0,2-1\%) de obstrucción intestinal alta. Se produce por la compresión de la tercera porción duodenal a su paso entre la arteria mesentérica superior y la aorta abdominal (compás aortomesentérico). Cuando el ángulo entre ambas estructuras disminuye y la distancia entre la aorta abdominal y la arteria mesentérica superior decrece, se produce la obstrucción duodenal ${ }^{1}$ (Figura 1). ${ }^{2}$ La tomografía computada con contraste endovenoso ha permitido confirmar su etiología. ${ }^{3}$

Es más común en pacientes de sexo femenino ( $2: 1)$ y en menores de 30 años. ${ }^{4}$ La etiopatogenia del SAMS puede deberse a causas congénitas o adquiridas. Por lo general, son adquiridas. La más frecuente es la pérdida brusca y grave de peso por crecimiento lineal sin incremento de peso en la adolescencia $\mathrm{u}$ otros síndromes consuntivos o estados catabólicos. Otras causas adquiridas son las que producen compresión extraabdominal, como posquirúrgicos de cirugías ortopédicas de escoliosis o inmovilizaciones que aumentan la lordosis lumbar. Las causas congénitas son el ligamento de Treitz corto y el nacimiento bajo de la arteria mesentérica superior. ${ }^{2,3}$

\section{CASO CLÍNICO}

Niña de 12 años que consultó por comenzar súbitamente con epigastralgia intensa y vómitos alimentarios reiterados. Presentaba antecedentes de una internación con igual clínica a los 10 años de edad, en la que se había diagnosticado gastritis secundaria a Helicobacter pylori y giardiasis. Dos años más tarde, la niña reinició la misma sintomatología: crisis de dolor abdominal y vómitos. 
Ingresó en regular estado general con facies dolorosa, eutrófica y con dolor en el epigastrio e hipocondrio derecho.

Durante la internación, se descartaron distintas causas de dolor abdominal en pediatría: gastritis, úlcera duodenal, colelitiasis, pancreatitis, trastornos en la alimentación, entre otros.

FIGURA 1. Ángulo aortomesentérico normal y en el sindrome de la arteria mesentérica superior que contiene, en su interior, la vena renal, el proceso uncinado del páncreas y la tercera porción del duodeno ${ }^{2}$

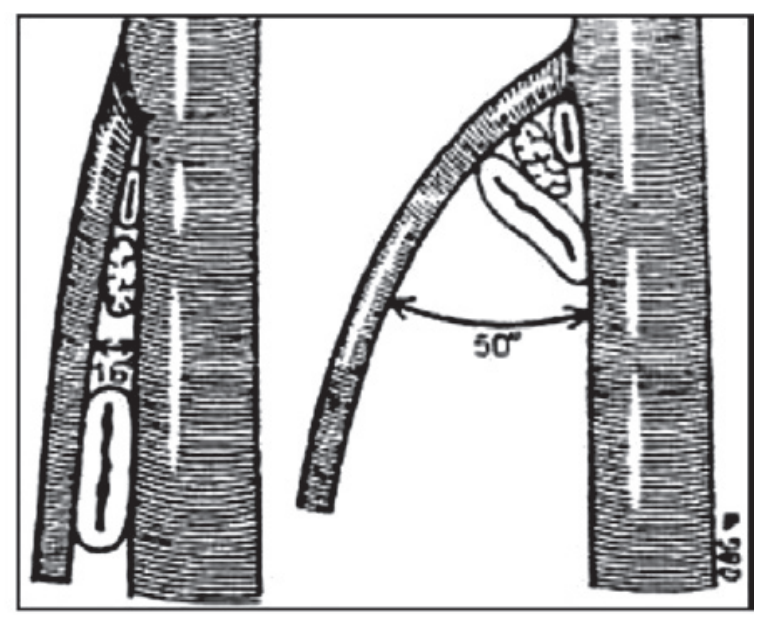

FIgURA 2. Angio tomografía computada de abdomen de la paciente (corte sagital). Se muestra el compás aortomesentérico disminuido $\left(20^{\circ}\right)$

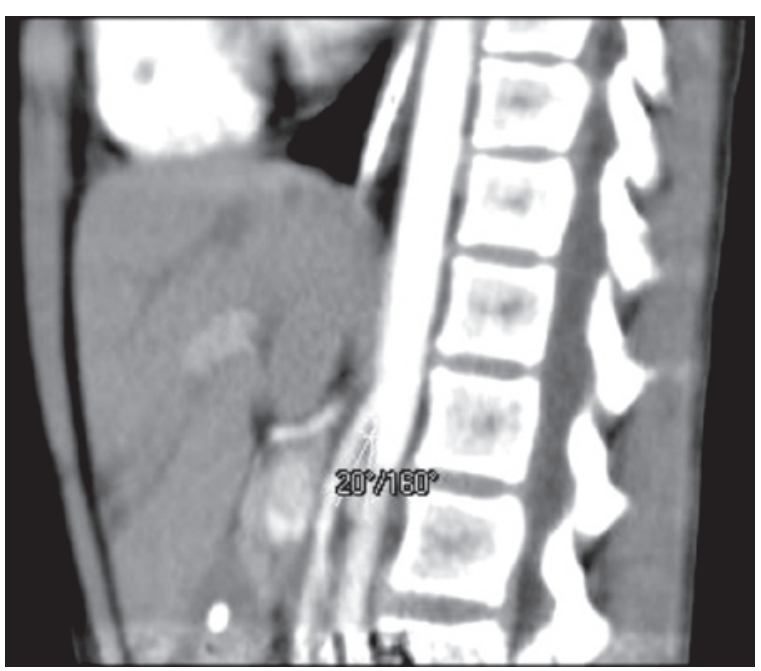

En el examen clínico diario de la niña, era llamativo el alivio del dolor posprandial en decúbito lateral izquierdo. A medida que la paciente perdía peso, las crisis de dolor y los vómitos aumentaban su frecuencia e intensidad con intervalos de tiempo asintomáticos cada vez más cortos.

Esta situación planteó la sospecha clínica de SAMS y se procedió a realizar una angio tomografía computada de abdomen, que buscaba medir el ángulo del compás aortomesentérico y la distancia aortoarteria mesentérica superior. Se observó la disminución de ambos en relación con los rangos normales: ángulo de $20^{\circ}$ y distancia de $3,8 \mathrm{~mm}$. Se corroboró la sospecha clínica del SAMS (Figura 2 y 3 ).

Se inició un tratamiento médico conservador, y se estabilizó a la paciente descomprimiendo el tracto gastrointestinal con la colocación de una sonda nasogástrica, la posición en decúbito lateral izquierdo posprandial, la medicación proquinética y antirreflujo, y el plan de recuperación nutricional con nutrición parenteral total y alimentación fraccionada por sonda nasogástrica.

A las tres semanas, debido a la persistencia de los vómitos, al aumento del dolor abdominal y a la incapacidad de recuperarse nutricionalmente, se consideró el fracaso del tratamiento médico y se decidió realizar el tratamiento quirúrgico.

Figura 3. Angio tomografía computada de abdomen de la paciente (corte axial). La medición de la distancia aortaarteria mesentérica superior se encuentra disminuida $(3,8 \mathrm{~mm})$

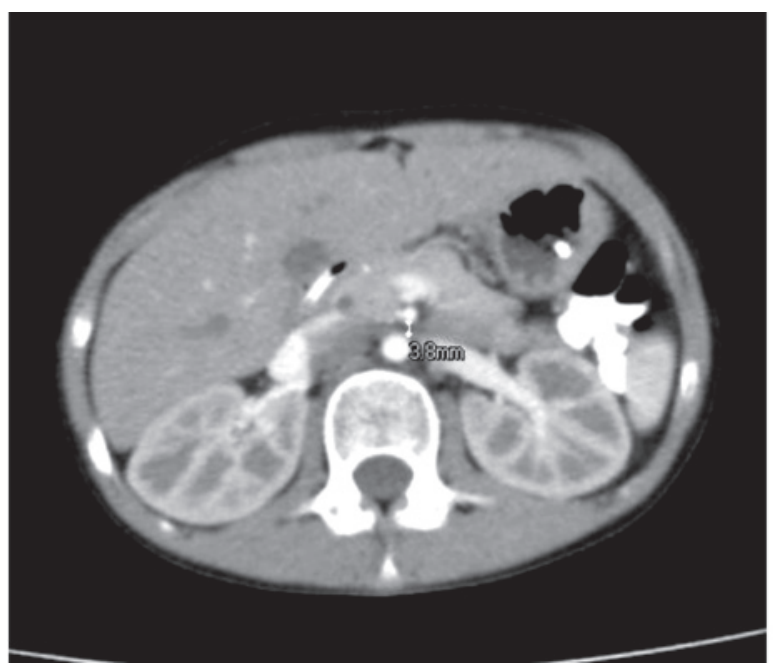


En primera instancia, se realizó una cirugía de Strong con el fin de liberar el ligamento de Treitz. Puesto que la paciente continuó con vómitos biliosos e intenso dolor abdominal, a los siete días, se volvió a intervenir y se realizó una gastroyeyuno-anastomosis.

No obstante esta segunda cirugía, la paciente continuó con igual sintomatología y no se evidenció ningún cambio favorable. A las tres semanas, se intervino otra vez y se realizó una duodenoyeyuno-anastomosis.

Luego de esta cirugía, se comenzó a observar la mejoría clínica. La niña aumentó de peso, disminuyeron los dolores abdominales y presentó una mejoría de su estado general. Se otorgó el alta hospitalaria luego de sesenta días posteriores al diagnóstico. Actualmente, luego de tres años, la paciente se encuentra asintomática, eutrófica y lleva una vida normal.

\section{DISCUSIÓN}

Los casos clínicos de SAMS que se reportan en pediatría, en su gran mayoría, tienen un factor predisponente; sin embargo, en nuestra paciente, no se encontró ninguno.

El espectro clínico es amplio, lo cual dificulta y alarga el proceso diagnóstico, por lo que requiere un alto índice de sospecha. Los síntomas pueden ser agudos, subagudos o crónicos y son secundarios a la obstrucción intestinal alta: dolor abdominal epigástrico posprandial que se alivia en posición genupectoral y en decúbito lateral izquierdo, vómitos biliosos, náuseas, distensión abdominal, saciedad precoz, sensación de plenitud gástrica y anorexia. ${ }^{5}$ Si hay compresión de la vena renal, el paciente puede presentar hipertensión arterial, hematuria o congestión venosa pelviana. ${ }^{6}$

El examen complementario de elección es la angio tomografía computada, en la que se puede medir el ángulo (rango normal: de $25^{\circ}$ a $50^{\circ}$ ) y la distancia disminuida a nivel de la tercera vértebra lumbar entre la arteria aorta abdominal y la mesentérica superior ${ }^{7}$ (rango normal: de 10 a $28 \mathrm{~mm}$ ). Estos parámetros de distancia y ángulo solo adquieren relevancia patológica cuando se acompañan de una clínica acorde a la entidad; en los pacientes asintomáticos, no tienen relevancia. También resulta de utilidad el estudio baritado, en el que se observa la dilatación gástrica y duodenal con interrupción abrupta o reducción de calibre a nivel de la tercera porción duodenal. La angio resonancia magnética nuclear (angioRMN) constituye un método diagnóstico de segunda línea.

El tratamiento inicial se centra en aliviar la obstrucción, mejorar la nutrición y corregir las anomalías hidroelectrolíticas asociadas. Colocar al paciente en decúbito lateral izquierdo o prono permite reanudar la ingestión oral. ${ }^{7}$ Lo esencial es prevenir la pérdida de peso que lleva a exacerbar los síntomas y lograr el peso adecuado para conservar el ángulo normal entre las dos arterias y evitar la recurrencia.

Más del $80 \%$ de los casos se resuelven con tratamiento médico; sin embargo, si este fracasa, la elección es la cirugía para derivar la obstrucción. Hay varias opciones quirúrgicas; la cirugía con duodeno-yeyunostomía laterolateral es exitosa en un $90 \%$ de los casos. ${ }^{9,10}$

\section{REFERENCIAS}

1. Fiorini S, Sáenz Tejeira M, Tennina C, Tomezzoli S, et al. Síndrome de la arteria mesentérica superior (Síndrome de Wilkie). Caso Clínico. Arch Argent Pediatr. 2008; 106(6);546-8.

2. Loja Oropeza D, Alvizuri Escobedo J, Vilca Vásquez M, Sánchez Mercado M. Síndrome de Wilkie: compresión vascular del duodeno. Rev Gastroenterol Perú. 2002; 22(3):248-52.

3. Ibarra F, Arraigada D. Síndrome de la arteria mesentérica superior: caso clínico y revisión. Boletín Escuela de Medicina UC, Pontificia Universidad Católica de Chile. 2006; 31(1):42-6.

4. Castaño Llano R, Chams Anturi A, Arango Vargas P, García Valencia A. Síndrome de la arteria mesentérica superior o síndrome de Wilkie. Rev Col Gastroenterol. 2009; 24(2):200-9.

5. Pastén González A, Muñoz Araneda A, Peirano Bastías A, Rojas Castro S, et al. Síndrome de la arteria mesentérica superior: Presentación de un caso y revisión de la literatura. Cir Pediatr. 2016; 29(4):180-3.

6. Rodríguez A, Romero Vidomlansky S, Ferrarotti C, Larrañaga $\mathrm{N}$, et al. Síndrome de la arteria mesentérica superior. Presentación de un caso. Rev Argent Radiol. 2014; 78(2):96-8.

7. Gutiérrez Junquera C, Gonzálvez Piñera J, Pérez Martínez A, Marco Macián A. Síndrome de la arteria mesentérica superior: una causa infrecuente de obstrucción duodenal en la infancia. An Esp Pediatr. 1996; 45(3):289-90.

8. Oliva Fonte C, Fernández Rey CL, Pereda Rodriguez J, González Fernández AM. Síndrome de Wilkie o pinza aortomesentérica. Rev Esp Enferm Dig. 2017; 109(1):62-3.

9. Ruiz Padilla FJ, Mostazo Torres J, Vílchez Jaime M. Gran distención gástrica en relación con síndrome de la arteria mesentérica superior o síndrome de Wilkie. Gastroenterol Hepatol. 2017; 40(8):581-3.

10. Biank V, Werlin S. Superior mesenteric artery syndrome in children: a 20 year experience. J Pediatr Gastroenterol Nutr. 2006; 42(5):522-5. 